\title{
Plain abdominal radiography in acute abdominal pain; past, present, and future
}

This article was published in the following Dove Press journal:

International Journal of General Medicine

12 June 2012

Number of times this article has been viewed

\author{
Sarah L Gans' \\ Jaap Stoker ${ }^{2}$ \\ Marja A Boermeester \\ 'Department of Surgery, \\ ${ }^{2}$ Department of Radiology, \\ Academic Medical Center, \\ University of Amsterdam, \\ Amsterdam, The Netherlands
}

Correspondence: Sarah L Gans Academic Medical Center, University of Amsterdam, Postbox 22660, II 00 DD, Amsterdam, The Netherlands Email s.l.gans@amc.uva.nl

\begin{abstract}
Several studies have demonstrated that a diagnosis based solely on a patient's medical history, physical examination, and laboratory tests is not reliable enough, despite the fact that these aspects are essential parts of the workup of a patient presenting with acute abdominal pain. Traditionally, imaging workup starts with abdominal radiography. However, numerous studies have demonstrated low sensitivity and accuracy for plain abdominal radiography in the evaluation of acute abdominal pain as well as various specific diseases such as perforated viscus, bowel obstruction, ingested foreign body, and ureteral stones. Computed tomography, and in particular computed tomography after negative ultrasonography, provides a better workup than plain abdominal radiography alone. The benefits of computed tomography lie in decision-making for management, planning of a surgical strategy, and possibly even avoidance of negative laparotomies. Based on abundant available evidence, major advances in diagnostic imaging, and changes in the management of certain diseases, we can conclude that there is no place for plain abdominal radiography in the workup of adult patients with acute abdominal pain presenting in the emergency department in current practice.
\end{abstract}

Keywords: abdominal x-ray, acute abdomen, acute abdominal pain, emergency department, diagnostic imaging, abdominal radiography

\section{Historical overview}

"Plain films are likely to remain the best method of imaging gas shadows for many years to come and computed tomography scanning, isotope studies and nuclear magnetic resonance are unlikely to play any major role in the initial investigation of the acute abdomen" (Field et $\mathrm{al}^{1}$ ).

Shortly after the discovery of the x-ray in 1895, the first x-rays were studied for medical purposes by Wilhelm Rontgen. During the decades that followed, $x$-rays were mainly used for detecting fractures and foreign bodies and gradually for the evaluation of various other diseases, such as acute abdominal pain.

Approximately $4 \%-10 \%$ of emergency department visits are due to acute abdominal pain, making it one of the most encountered complaints. ${ }^{2}$ The wide variety in presentation of symptoms and the broad spectrum of associated diseases complicates isolation of the cause of abdominal pain, which may vary from life-threatening diseases requiring emergency surgery to mild self-limiting causes. ${ }^{2}$ An early and accurate diagnosis is essential in decision-making, and insufficient workup results in unnecessary interventions or delayed treatment. ${ }^{2-4}$

A study performed at the University of Virginia in the US compared data from patients with acute abdominal pain at three different time points over a period 
of 35 years, ie, 1972, 1992, and $2007 .^{5}$ The proportion of patients presenting with acute abdominal pain as a chief complaint remained more or less stable over the years, being $4 \%$ in $1972,5 \%$ in 1992 , and $6.6 \%$ in 2007.

Plain abdominal radiography was the only diagnostic imaging modality available in 1972 and was ordered in $43 \%$ of all patients. In 1992, plain abdominal radiography was ordered in $30 \%$ of all patients. Ultrasound and computed tomography (CT) had come into use but were barely ordered, ie, in only $6.8 \%$ of all patients. In 2007, the use of ultrasound and CT was widespread and the use of plain abdominal radiography decreased somewhat, but was still performed in a considerable proportion of patients (21\%). CT and ultrasound were used liberally, and either one of these tests was performed in $42 \%$ of all patients. These data show increased use of CT and ultrasound and decreased use of plain abdominal radiographs (a decrease of approximately one third) between 1992 and 2007.

In the same time period, the average time patients spent at the emergency department had risen from 2.9 hours in 1992 to 4.26 hours in 2007. Patients who had undergone a CT scan spent an average of 6.64 hours in the emergency department compared with 3.44 hours without CT evaluation.

Diagnostic accuracy improved over the years due to increased use of CT and ultrasound; in 1992, 41.3\% of all patients were diagnosed with nonspecific abdominal pain compared with $21.1 \%$ in 2007 . The number of patients admitted to the ward decreased from $27.4 \%$ in 1972 to $18.4 \%$ in 1992.

Factors contributing to improved diagnostic accuracy include establishment of emergency medicine faculties and increased possibilities for laboratory testing. The widespread availability of various diagnostic modalities, such as CT, ultrasound, and nuclear imaging, probably account for the most significant change..$^{5-7}$

Diagnosing the underlying cause of acute abdominal pain remains a challenge despite the increase in diagnostic accuracy over the years. The ideal diagnostic imaging modality for evaluation of acute abdominal pain in adult patients at the emergency department should provide a balance between the highest diagnostic value and most accurate management changes on one hand, and lowest radiation exposure, discomfort, and duration of stay at the emergency department on the other hand, while ultimately resulting in the lowest cost to the health care system.

\section{Place of plain abdominal x-ray in current diagnostic workup}

Several studies have demonstrated that a diagnosis based solely on a patient's medical history, physical examination, and laboratory tests is not reliable enough, despite the fact that these aspects are essential parts of the workup of a patient presenting with acute abdominal pain. ${ }^{2,8}$ Further diagnostic workup such as imaging is therefore mandatory in patients suspected of an urgent medical condition.

Imaging workup traditionally starts with abdominal radiography. ${ }^{9}$ Standard abdominal radiography consists of three views, ie, a supine abdominal view combined with an erect chest film and an upright abdominal view. ${ }^{10,11}$ UK and US guidelines advise consideration of abdominal radiography in case of hospital admission and or surgery in patients presenting with acute abdominal pain. ${ }^{12,13}$

One study compared the initial diagnosis after clinical evaluation and plain radiography with the final diagnosis, and found that these diagnoses corresponded in only 502 of a total of 1021 patients (49\%). ${ }^{3}$ Diagnosis based only on plain abdominal radiography corresponded with the final diagnosis in 514 of the 1021 patients $(50 \%)$. The improvement in accuracy of plain abdominal radiography combined with clinical examination was not significant $(P=0.14)$. In 117 of the 1021 patients $(11 \%)$, the treating physician changed the initial clinical diagnosis after plain abdominal radiography, and these changes were accurate in only 39 cases (22\%). Additionally, the level of confidence of the assigned diagnosis was recorded in 983 patients before and after plain abdominal radiography. For 875 patients in whom the diagnosis had not changed, the level of confidence also remained unchanged.

Several studies have demonstrated a high percentage of plain abdominal radiographs without abnormal or specific findings. Two studies demonstrated that $77 \%$ and $78 \%$ of all requested plain abdominal radiographs showed no abnormal findings. ${ }^{14,15}$ Another study demonstrated that in only 83 of 871 patients $(10 \%)$, a specific diagnostic abnormality was noted on plain abdominal radiography. ${ }^{16}$

A study analyzing the value of plain abdominal radiography in addition to clinical examination showed that management changed in only 15 patients (8.9\%). ${ }^{17}$ In 90 of 153 remaining patients $(53.6 \%)$, the initial diagnosis changed due to other imaging modalities, and in 63 patients the diagnosis remained unchanged after plain abdominal radiography.

Whether plain abdominal radiography contributes to therapeutic decision-making or disposition remains questionable. Particularly in the case of a negative result, the additional value of plain abdominal radiographs is disputed. It is for this reason that several studies suggest ordering plain abdominal radiographs for specific indications only, in order to reduce the number of unnecessary requests. ${ }^{11-13,18,19}$ Specific indications for ordering plain radiography include 
suspicion of perforated viscus, urinary tract stones, bowel obstruction, and ingested foreign body.

\section{Detection of free air and perforated viscus}

"The identification of a small amount of free intra-abdominal gas remains one of the most significant signs in medicine.
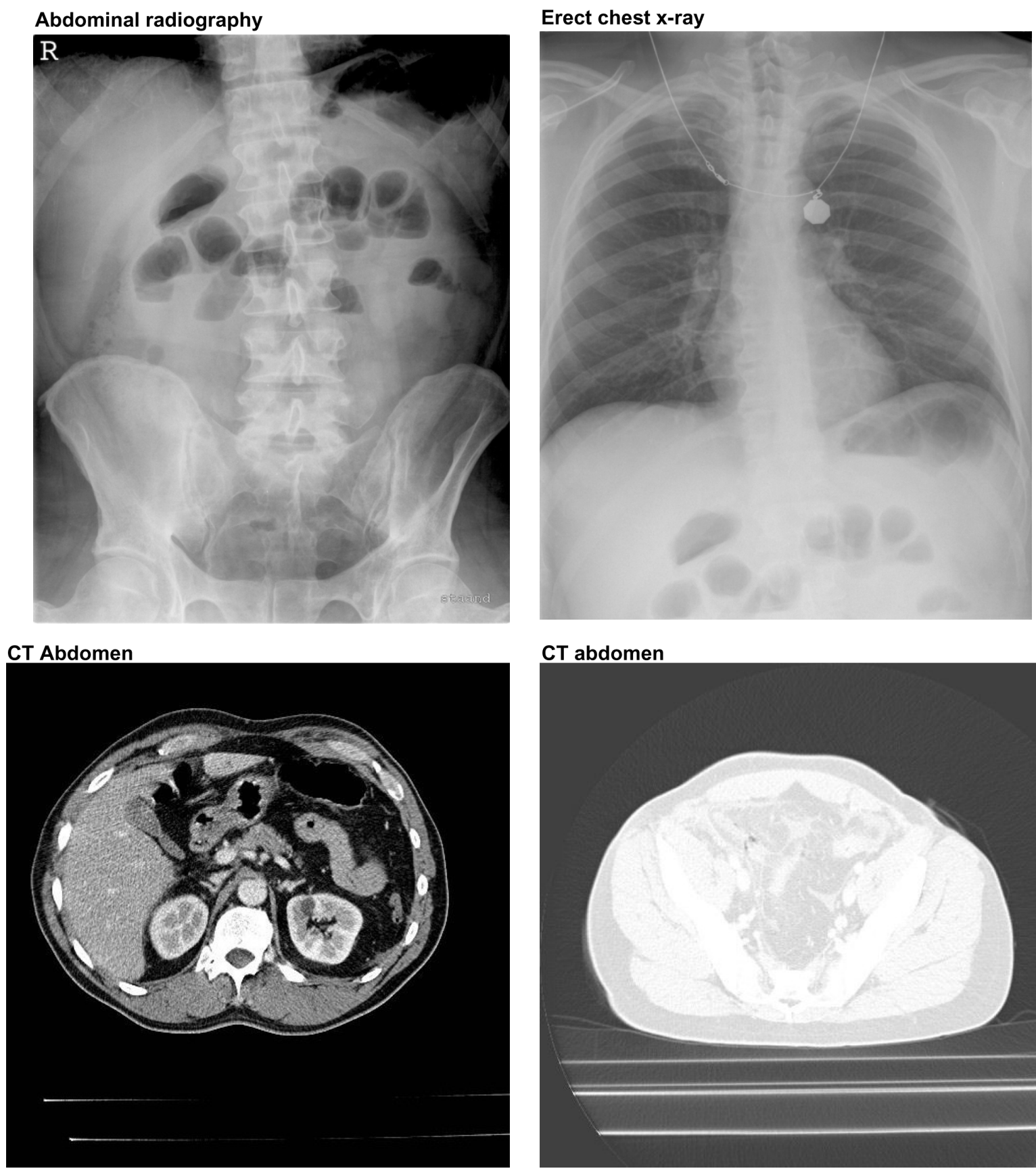

\section{CT abdomen}

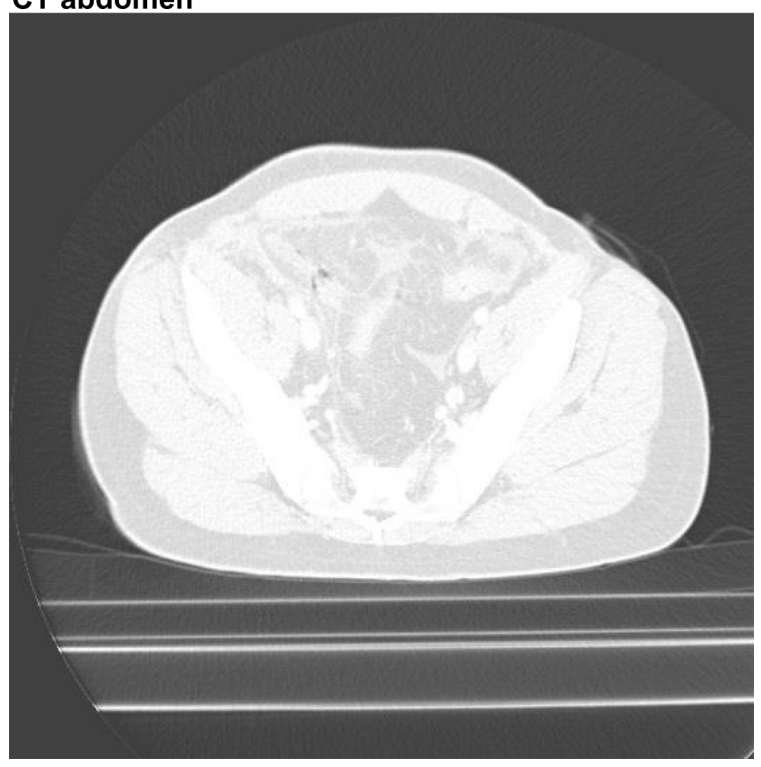

Case I Free air.

Notes: A 48-year-old male presented at the emergency department with pain in the entire abdomen, but concentrating in the right lower quadrant. Palpation of the entire abdomen was extremely painful and laboratory values showed elevated inflammatory parameters (leucocyte count 17.9 and C-reactive protein 43). Upright abdominal radiography showed no abnormalities. Computed tomography of the abdomen showed free intraperitoneal air and signs of appendicitis acuta. Patient underwent an emergency laparotomy, which confirmed the diagnosis of perforated appendicitis acuta. 
careful radiographic technique, to demonstrate as little as $1 \mathrm{~mL}$ of free gas on an erect chest or left lateral decubitus abdominal film. ${ }^{20}$ The high percentage of missed cases is due to technical imperfections rather than limitations of the test (poor quality of plain abdominal radiography, excluding the uppermost portion of the peritoneal cavity of the image). ${ }^{21}$ In that study, the radiographs demonstrated pneumoperitoneum in only $51 \%$ of patients with documented visceral perforation. Diagnostic accuracy differed between the types of radiograph used to demonstrate pneumoperitoneum. Left lateral decubitus radiographs demonstrated pneumoperitoneum in $96 \%$ of patients, chest radiographs in $85 \%$, and upright and supine abdominal radiographs in $60 \%$ and $56 \%$, respectively. ${ }^{22}$ Another study described pneumoperitoneum in only $83 \%$ of all patients with documented visceral perforation. ${ }^{23}$

One study compared the diagnosis of patients suspected of perforated viscus before and after plain abdominal radiography. ${ }^{3}$ The positive predictive value was not influenced by plain abdominal radiography. Sensitivity of abdominal radiography in detecting pneumoperitoneum was low $(15 \%)$. The level of confidence in the diagnosis changed in six of $13(46 \%)$ patients with the clinical diagnosis of a perforated viscus. These data were obtained from a cohort of 1021 patients who presented at the emergency department with acute abdominal pain. ${ }^{2}$ This study demonstrates that plain abdominal radiographs have no added value in the diagnostic workup.

Several studies have demonstrated that plain abdominal radiography has a lower accuracy than other diagnostic modalities. One study compared ultrasonography with plain abdominal radiography in detection of pneumoperitoneum, and included 188 patients suspected of visceral perforation. ${ }^{24}$ All patients underwent chest and/or abdominal radiography and ultrasonography in order to detect free intraperitoneal air; 165 patients had suspicion of pneumoperitoneum after ultrasonography, and in 157 of the suspected patients, visceral perforation was confirmed intraoperatively. After plain radiography, 126 patients were suspected of pneumoperitoneum which was confirmed intraoperatively in 120 of cases.

Both diagnostic modalities demonstrated high positive predictive value (95\% in ultrasound and 94\% in radiography) and similar specificity (53\%). Ultrasound did have a higher sensitivity ( $92 \%$ versus $78 \%$ ), accuracy ( $88 \%$ versus $76 \%$ ), and negative predictive value (39\% versus $20 \%$ ).

CT has proven to be the most accurate diagnostic modality in the evaluation of pneumoperitoneum. ${ }^{25} \mathrm{~A}$ small study compared CT with plain radiographic evaluation in 13 patients who underwent diagnostic peritoneal lavage due to abdominal trauma. ${ }^{25}$ Only five of 13 patients $(38 \%)$ demonstrated free air on radiographs compared with 13 of 13 patients on CT. Another study retrospectively reviewed $\mathrm{CT}$ and plain radiographs (when available) of 76 patients with proven perforation of the gastrointestinal tract. ${ }^{26}$ In 65 of 76 patients, CT was truly positive and in 11 patients was falsely negative. The cause of perforation was correctly predicted in $51(78 \%)$ of all patients, and the location of the perforation was correctly predicted in 55 patients $(84.6 \%)$ on CT. In 63 patients plain radiographs were used, of which 32 (52\%) were truly positive and 31 (48\%) were falsely negative for the presence of a perforation.

The available evidence demonstrates that even though the positive predictive value of plain abdominal radiography is similar to that of other diagnostic modalities, the sensitivity and negative predictive value are far too low. The additional value of CT lies in the possibility of providing more information on the location and underlying cause of the perforation, or in providing an alternative diagnosis. In current practice, the high number of missed cases of perforation after plain abdominal radiography is unacceptable and renders the additional value of negative plain abdominal radiography very limited. Moreover, a plain abdominal radiograph that is positive for free air conveys limited information on the location and underlying cause; an additional CT scan will often need to be made to be able to adapt the operative strategy to the specific case. Performing surgery without adequate information about the perforation site is a conceptual mistake and nowadays should be considered as substandard clinical practice (not all free air is caused by a perforated gastric or duodenal ulcer; consider, amongst others, colonic perforation, surgery-related perforation, perforated diverticular disease, perforated appendicitis, inflammatory bowel disease, or endoscopy). No place exists for abdominal radiography in the evaluation of patients suspected of visceral perforation or, for that matter, in any patient with acute abdominal pain.

\section{Detection of urinary tract stones}

The standard imaging modality for detecting urinary tract stones should ideally provide information about size, site, and composition of the ureteral stone and presence of ureteral obstruction.

Most ureteral stones can be identified as a calcification causing a filling defect or ureteral obstruction on plain abdominal radiography. ${ }^{1}$ A plain film of the abdomen, including kidney, ureter, and bladder, has shown sensitivity ranging from $44 \%$ up to $77 \%$ and specificity in detection of stones from $80 \%$ to $87 \% .{ }^{27-29}$ 

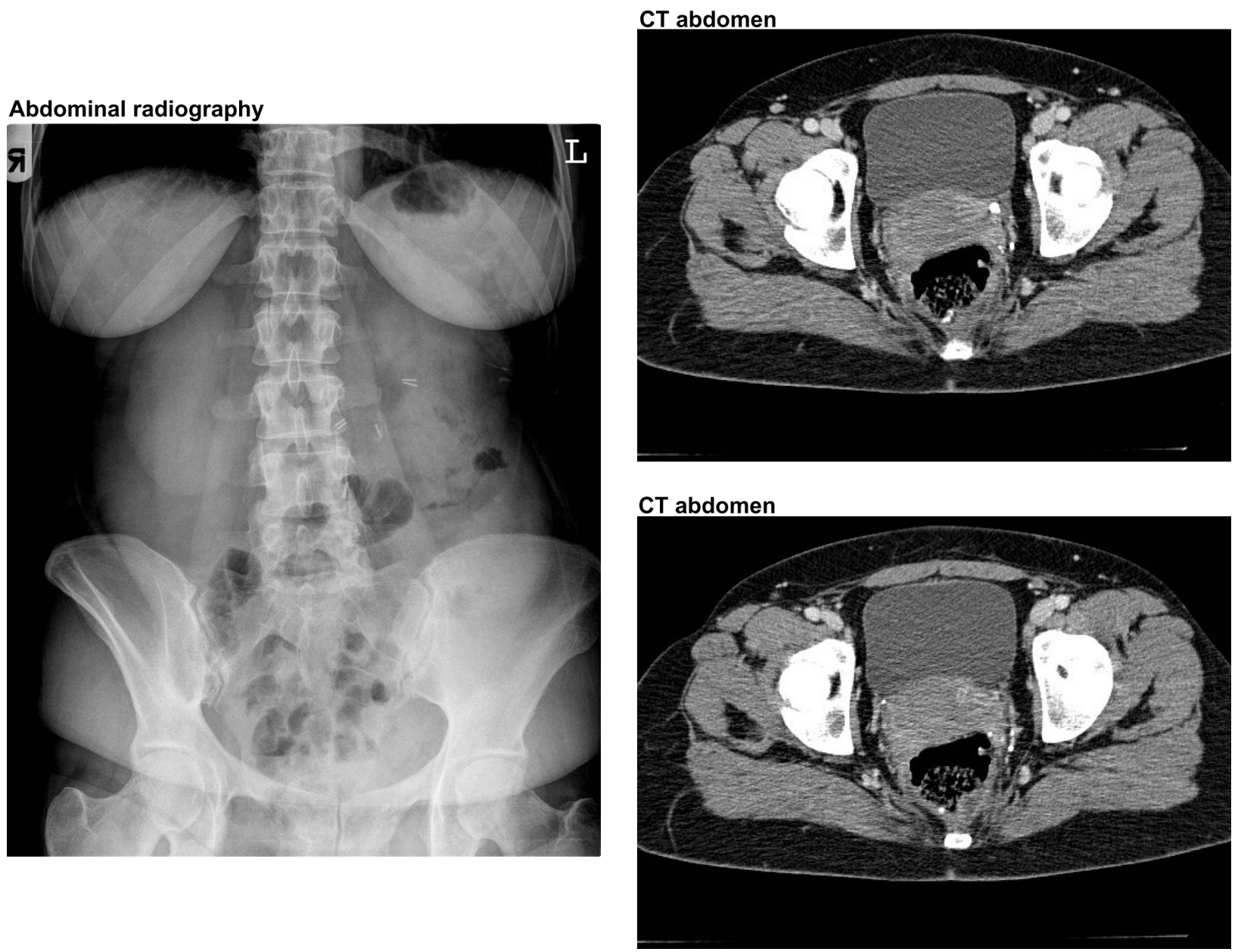

Case 2 Urinary tract stones.

Notes: A 36-year-old female presented at the emergency department with left-sided abdominal pain over the course of 6 hours. Laboratory values showed elevated inflammatory parameters (leucocyte count 15.3 and C-reactive protein 44). Based on clinical examination, the patient was suspected of having bowel obstruction or kidney stones, and an abdominal radiograph was ordered. Abdominal radiography demonstrated no abnormalities other than multiple clips related to previous bowel surgery. Computed tomography of the abdomen demonstrated hydronephrosis and signs of pyelonephritis of the left kidney due to an obstructing ureteral stone.

In one study, the diagnosis of patients suspected of ureteral stones before and after plain abdominal radiography compared with the final diagnosis revealed a correct change in diagnosis in six of 11 patients $(55 \%) .{ }^{3}$ The level of confidence in the diagnosis remained the same as well as having a positive predicting value, which reached $57 \%$ after clinical evaluation and $58 \%$ after radiographs.

Another study concluded that plain abdominal radiography had a sensitivity of $45 \%$ and specificity of $77 \%$ for detection of ureteral calculi. ${ }^{30}$ In patients previously diagnosed with urolithiasis presenting with similar symptoms, abdominal radiography could be useful to diagnose kidney stones. The advantage of CT over plain abdominal radiography is that an alternative diagnosis may be presented if the suspicion of urolithiasis is not confirmed. Additional information concerning urolithiasis may also be obtained by $\mathrm{CT}$, such as size and location, which are both crucial factors in guiding therapy. CT has replaced the use of plain abdominal radiography and intravenous urography in the detection of ureteral stones.

\section{Detection of bowel obstruction}

Bowel obstruction is a common cause of acute abdominal pain. About $7 \%$ of all patients with acute abdominal pain are diagnosed with bowel obstruction. Imaging in patients suspected of bowel obstruction should provide information about the site, cause, and level of the obstruction. ${ }^{31}$

Plain abdominal radiography is advocated as a standard diagnostic modality for the detection of bowel obstruction. ${ }^{12,13}$ Plain abdominal radiographic findings have been shown to be diagnostic in $50 \%-60 \%$, indifferent in $20 \%-30 \%$, and misleading in $10 \%-20 \%$ of patients. ${ }^{32}$

In one study, the sensitivity of plain abdominal radiography after clinical evaluation was significantly higher 

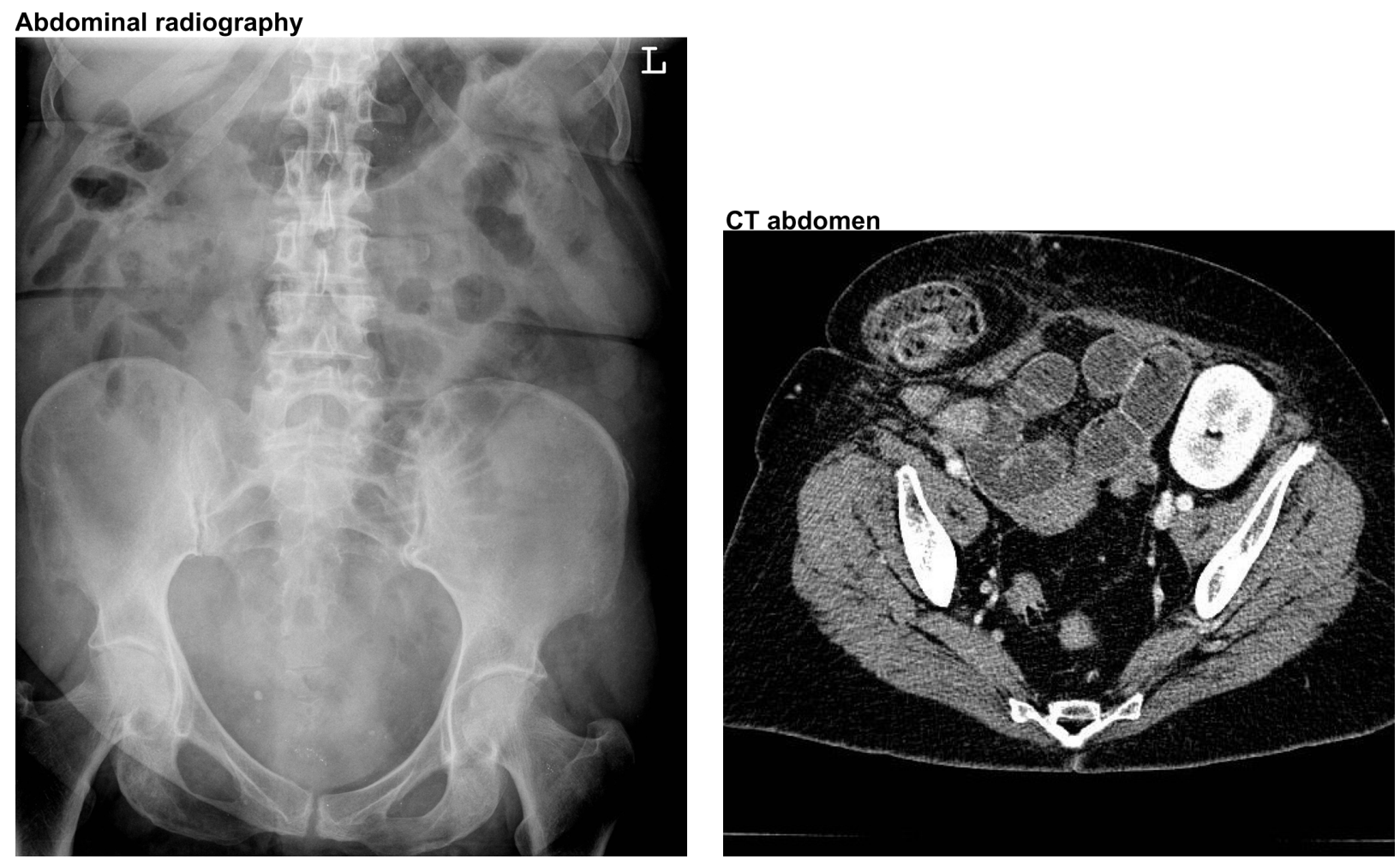

Case 3 Bowel obstruction.

Notes: A 59-year-old female presented at the emergency department with complaints of nausea, vomiting, and abdominal pain for one day. Physical examination demonstrated abdominal tenderness in all quadrants. Laboratory values were within normal limits, with the exception of slightly raised inflammatory parameters (C-reactive protein I7, leucocyte count 8) The attending physician suspected a bowel obstruction and ordered an abdominal radiograph. Abdominal radiography showed no abnormalities in addition to minimal dilation of the small bowel. Computed tomography demonstrated dilated small bowel loops, collapsed large bowel loops, and a change in diameter due to a herniation of small bowel into the right musculus rectus abdominus. Images were suggestive of an incarcerated herniation. After reduction of herniation at the emergency department, her complaints resolved and she made an uneventful recovery.

than that of clinical evaluation alone ( $74 \%$ versus $57 \%$, respectively). ${ }^{3}$ Changes in diagnosis after plain abdominal radiography were correct in only 16 of 24 patients (66\%) and the level of confidence remained unchanged in 32 of 71 patients (52\%). The sensitivity of a clinical evaluation combined with a score card for clinical signs/symptoms proved to be similar to a clinical evaluation combined with plain abdominal radiography. ${ }^{3}$

Frager et al compared diagnoses after clinical evaluation combined with plain abdominal radiography or $\mathrm{CT} .^{33} \mathrm{In}$ patients with a complete obstruction, CT demonstrated a sensitivity of $100 \%$ compared with $46 \%$ after plain abdominal radiography. For partial obstruction, CT had a sensitivity of $100 \%$ compared with $30 \%$ for plain abdominal radiography. Of the 61 patients who underwent surgery, 52 patients were confirmed to be correctly diagnosed preoperatively (85\%) based on CT findings. The exact location of the obstruction was correctly diagnosed in 50 of 53 patients (94\%) on CT.

The diagnostic accuracy of CT is superior to that of plain abdominal radiography. In addition to its higher sensitivity, an important advantage of $\mathrm{CT}$ is the ability to provide information about the underlying cause of obstruction or to provide information about an alternative diagnosis if no signs of bowel obstruction are present. CT leads to more accurate management and assistance in preoperative planning.

\section{Detection of ingested foreign body}

Diagnostic imaging is not always necessary in cases of ingested foreign bodies. Additional diagnostic investigations should be considered if clinically relevant, particularly if surgery is considered. Most foreign bodies pass through the gastrointestinal tract harmlessly. If patients are symptomatic or if the ingested foreign body is potentially dangerous, additional diagnostic examinations should be undertaken.

Plain radiography has been suggested as a standard method for localization of foreign bodies. Plain abdominal radiography demonstrates a sensitivity of $90 \%$, specificity of $100 \%$, and accuracy of $100 \%$ for ingested foreign bodies, but the foreign body has to be radio-opaque to be seen on plain abdominal radiography. ${ }^{16}$ There is no evidence available as to whether CT has a higher sensitivity and specificity than plain 

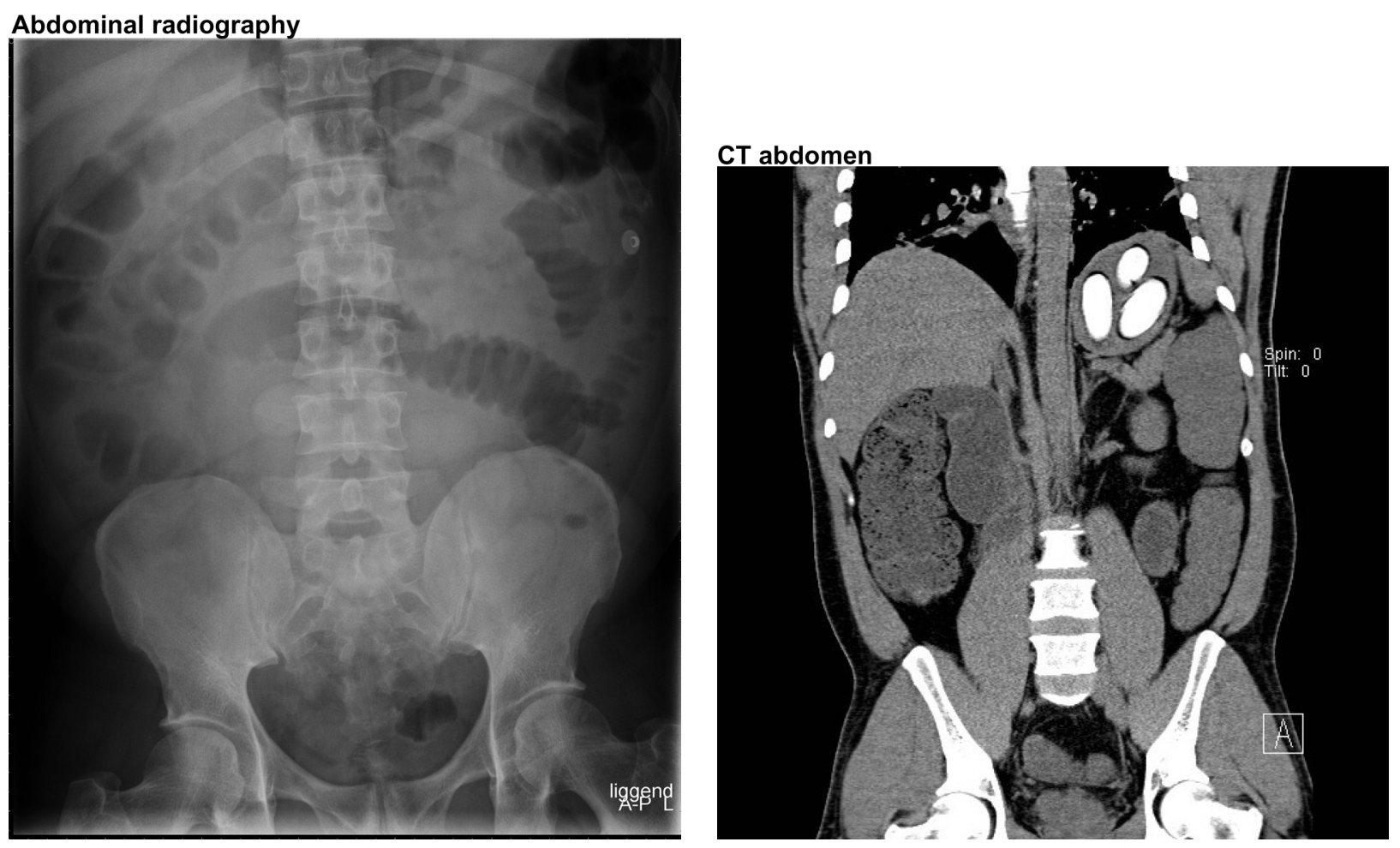

Case 4 Ingested foreign body.

Notes: A 35-year-old male presented at the emergency department with acute abdominal pain, tachycardia, and a diffusely rigid abdomen. The patient admitted having ingested eight packets of drugs three days earlier. An abdominal radiograph was done to confirm ingestion of the packets and to clarify the location and exact number of packets in need of surgical removal. At least four packets were identified on abdominal radiographs and the patient underwent a laparotomy due to signs of intoxication; eight packets of drugs were identified and surgically removed from the small bowel. Postoperatively, the patient remained tachycardic and in pain; a computed tomography scan was done 24 hours after the initial laparotomy, showing an additional five packets of drugs in the stomach and ileum.

abdominal radiography in the case of ingested foreign bodies. The advantage of CT is the ability to provide information about the location of the foreign body, which is a prerequisite when surgical treatment is planned.

Body packers form a specific category of ingested foreign bodies. Body packers orally ingest, or rectally or vaginally insert packed drugs in order to smuggle them. Plain abdominal radiography is used to establish the diagnosis of drug packing and is considered the gold standard. ${ }^{34}$ If plain abdominal radiography is negative or inconclusive but a high suspicion of body packing remains, a CT scan should be done. The sensitivity of plain abdominal radiography is $85 \%-100 \%$, but CT has a higher sensitivity and additionally provides more accurate information about the number and location of packages. The use of plain abdominal radiography gives rise to a high number of false negative results, which could be due to overprojection of feces or a specific packaging method. ${ }^{35}$

There is no solid evidence that CT has a higher sensitivity or diagnostic accuracy than plain abdominal radiography. The value of CT lies in preoperative planning. The low negative predictive value of plain abdominal radiography leads to the conclusion that, if clinically relevant, a CT should be used as the diagnostic modality of choice.

\section{In diagnostic workup}

"In spite of the recent proliferation of other imaging techniques, plain films still retain their position as one of the most useful initial investigations" (Field et $\mathrm{al}^{1}$ ).

Imaging techniques such as $\mathrm{CT}$ and ultrasound have been shown to increase diagnostic accuracy substantially, ${ }^{5}$ and consequently have significantly decreased the added diagnostic value of plain abdominal radiography in a clinical setting. Despite recent abundant evidence of its limited value, many physicians still rely on plain abdominal radiography as a simple, cheap, and widely available first diagnostic modality with lower radiation exposure than CT. Proponents of plain abdominal radiography advocate its use to prevent high radiation exposure in patients due to unnecessary CT imaging. The average plain abdominal radiograph exposes the patient to $0.7 \mathrm{mSv}$ and an abdominal CT exposes the patient to $10.0 \mathrm{mSv} \cdot{ }^{13,36} \mathrm{New}$ techniques, such as automated dose modulation and an iterative reconstruction algorithm, reduce the CT radiation dose. 
In everyday clinical practice, physicians differentiate, subconsciously or consciously, between urgent and nonurgent conditions in patients. Patients with indeterminant symptoms and low or no suspicion of an urgent condition can be discharged home without additional imaging. Patients suspected of urgent conditions require timely identification of the underlying cause and treatment within 24 hours to prevent severe complications.

A recent study designed to identify the most effective diagnostic strategy for patients with acute abdominal pain has demonstrated that the highest sensitivity for detecting urgent diagnoses is achieved when ultrasonography is performed in all patients and a CT only in the event of inconclusive or negative ultrasonography (conditional CT strategy). ${ }^{31}$ Using this strategy, CT is only needed in $49 \%$ of patients.

Even though CT exposes patients to a higher radiation dose, it still remains the preferred standard diagnostic modality if ultrasound fails to diagnose the cause of acute abdominal pain correctly. Plain radiography demonstrates low sensitivity and accuracy and is therefore generally unhelpful; because of its low sensitivity and negative predictive value, it can also be misleading in the workup of acute abdominal pain. Although the radiation dose is lower than that of CT, the mediocre test characteristics of plain radiography may delay appropriate treatment and are therefore detrimental.

Numerous studies have demonstrated low sensitivity and accuracy for plain abdominal radiography in the evaluation of acute abdominal pain as well as for various specific diseases, such as perforated viscus, bowel obstruction, ingested foreign body, and ureteral stones.

CT provides a better workup than that achieved with plain abdominal radiography alone, and its benefits lie in improving decision-making for management, adapting the surgical strategy, and possibly even avoiding negative laparotomies.

When a new test is developed, it can have three possible roles in relation to the existing situation. The existing test can be replaced, the new test could be added on top of the existing test, or the new test could function as a triage test to distinguish between patients who need further examination and those who can be safely observed without additional examination.

If we were to look at abdominal radiography as if it was a newly developed test for adult patients with acute abdominal pain in the emergency department, the evidence presented in this manuscript demonstrates that there is no added value nor could abdominal radiography replace ultrasound or CT.
If abdominal radiography were to be used as triage, its main purpose would be to rule out disease in patients who genuinely do not have disease, ie, to prevent further investigations. However, for this purpose, the number of false negatives has to be relatively low and sensitivity should be high, which is not the case for abdominal radiographs. ${ }^{37}$

Based on the abundant available evidence, major advances in diagnostic imaging, and changes in the management of certain diseases, we can conclude that there is no place for plain abdominal radiography in the workup of adult patients with acute abdominal pain in current practice.

\section{Disclosure}

The authors report no conflicts of interest in this work.

\section{References}

1. Field S. Plain films: the acute abdomen. Clin Gastroenterol. 1984;13(1): 3-40.

2. Lameris W, van Randen A, van Es HW, et al. Imaging strategies for detection of urgent conditions in patients with acute abdominal pain: diagnostic accuracy study. Br Med J. 2009;338:b2431.

3. van Randen A, Laméris W, Luitse JSK, et al. The role of plain radiographs in patients with acute abdominal pain at the ED. Am J Emerg Med. 2011;29(6):582-589.

4. MacKersie AB, Lane MJ, Gerhardt RT, et al. Nontraumatic acute abdominal pain: unenhanced helical CT compared with three-view acute abdominal series. Radiology. 2005;237(1):114-122.

5. Hastings RS, Powers RD. Abdominal pain in the ED: a 35 year retrospective. Am J Emerg Med. 2011;29(7):711-716.

6. Brewer RJ, Golden GT, Hitch DC, Rudolf LE, Wangensteen SL. Abdominal pain: an analysis of 1000 consecutive cases in a university hospital emergency room. Am J Surg. 1976;131(2):219-223.

7. Kamin RA, Nowicki TA, Courtney DS, Powers RD. Pearls and pitfalls in the emergency department evaluation of abdominal pain. Emerg Med Clin North Am. 2003;21(1):61-72.

8. Navarro Fernández JA, Tárraga López PJ, Rodríguez Montes JA, López Cara MA. Validity of tests performed to diagnose acute abdominal pain in patients admitted at an emergency department. Rev Esp Enferm Dig. 2009;101(9):610-618.

9. Karkhanis S, Medcalf J. Plain abdomen radiographs: the right view? Eur J Emerg Med. 2009;16(5):267-270.

10. Flak B, Rowley VA. Acute abdomen: plain film utilization and analysis. CanAssoc Radiol J. 1993;44(6):423-428.

11. Greene CS. Indications for plain abdominal radiography in the emergency department. Ann Emerg Med. 1986;15(3):257-260.

12. Royal College of Radiologists. Referral guidelines for imaging. http:// ec.europa.eu/energy/nuclear/radioprotection/publication/doc/118_ en.pdf 2000: 1-127.

13. American College of Radiologists. Practice guideline for the performance of abdominal radiography. Radiography. 2006:1-5.

14. Lacey GJD, Wignall BK, Bradbrooke S, et al. Rationalising abdominal radiography in the accident and emergency department. Clin Radiol. 1980;31(4):453-455.

15. McCook TA, Ravin CE, Rice RP. Abdominal radiography in the emergency department: a prospective analysis. Ann Emerg Med. 1982; 11(1):7-8.

16. Ahn SH, Mayo-Smith WW, Murphy BL, Reinert SE, Cronan JJ. Acute nontraumatic abdominal pain in adult patients: abdominal radiography compared with CT evaluation. Radiology. 2002;225(1):159-164.

17. Prasannan S, Zhueng TJ, Gul YA. Diagnostic value of plain abdominal radiographs in patients with acute abdominal pain. Asian J Surg. 2005; 28(4):246-251. 
18. Hayward MW, Hayward C, Ennis WP, Roberts CJ. A pilot evaluation of radiography of the acute abdomen. Clin Radiol. 1984;35(4):289-291.

19. Anyanwu AC, Moalypour SM. Are abdominal radiographs still overutilized in the assessment of acute abdominal pain? A district general hospital audit. JR Coll Surg Edinb. 1998;43(4):267-270.

20. Miller RE, Nelson SW. The roentgenologic demonstration of tiny amounts of free intraperitoneal gas: experimental and clinical studies. Am J Roentgenol Radium Ther Nucl Med. 1971;112(3):574-585.

21. Baker SR. Unenhanced helical CT versus plain abdominal radiography: a dissenting opinion. Radiology. 1994;204(3):45-47.

22. Roh JJ, Thompson JS, Harned RK, Hodgson PE. Value of pneumoperitoneum in the diagnosis of visceral perforation. Am J Surg. 1983; 146(6):830-833.

23. Keefe EJ, Gagliardi RA. Significance of ileus in perforated viscus. $A m$ J Roentgenol Radium Ther Nucl Med. 1973;117(2):275-280.

24. Romero JA, Castaño N. Ultrasonography is superior to plain radiography in the diagnosis of pneumoperitoneum. Br J Surg. 2002; 89(3):351-354.

25. Stapakis JC, Thickman D. Diagnosis of pneumoperitoneum: abdominal CT vs upright chest film. J Comput Assist Tomogr. 1992;16(5): 713-716.

26. Maniatis V, Chryssikopoulos H, Roussakis A, et al. Perforation of the alimentary tract: evaluation with computed tomography. Abdom Imaging. 2000;25(4):373-379.

27. Mutgi A, Williams JW, Nettleman M. Renal colic: utility of the plain abdominal roentgenogram. Arch Intern Med. 1991;151(8): 1589-1592.

28. Roth CS, Bowyer BA, Berquist TH. Utility of the plain abdominal radiograph for diagnosing ureteral calculi. Ann Emerg Med. 1985;14(4):311-315.
29. Yilmaz S, Sindel T, Arslan G, et al. Renal colic: comparison of spiral CT, US and IVU in the detection of ureteral calculi. Eur Radiol. 1998;8(2): 212-217.

30. Levine MS. Plain film diagnosis of the acute abdomen. Emerg Med Clin North Am. 1985;3(3):541-562.

31. Lameris W, Van Randen A, Van Es HW, et al. Imaging strategies for detection of urgent conditions in patients with acute abdominal pain: diagnostic accuracy study. Br Med J. 2009;338:b2431.

32. Maglinte DT, Reyes BL, Harmon BH, et al. Reliability and role of plain film radiography and CT in the diagnosis of small-bowel obstruction. AJR Am J Roentgenol. 1996;167(6):1451-1455.

33. Frager D, Medwid SW, Baer JW, Mollinelli B, Friedman M. CT of smallbowel obstruction: value in establishing the diagnosis and determining the degree and cause. AJR Am J Roentgenol. 1994;162(1):37-41.

34. Niewiarowski S, Gogbashian A, Afaq A, Kantor R, Win Z. Abdominal $\mathrm{x}$-ray signs of intra-intestinal drug smuggling. J Forensic Leg Med. 2010;17(4):198-202.

35. van Geloven AA, van Lienden KP, Gouma DJ. Bodypacking - an increasing problem in The Netherlands: conservative or surgical treatment? Eur J Surg. 2002;168(7):404-409.

36. Wall BF, Hart D. Revised radiation doses for typical X-ray examinations. Report on a recent review of doses to patients from medical X-ray examinations in the UK by NRPB. National Radiological Protection Board. Br J Radiol. 1997;70(833):437-439.

37. Leeflang M, Deeks J, Gatsonis C. Systematic reviews of diagnostic test accuracy. Ann Intern Med. 2008;149(12):889-897.
International Journal of General Medicine

\section{Publish your work in this journal}

The International Journal of General Medicine is an international peer-reviewed open-access journal that focuses on general and internal medicine, pathogenesis, epidemiology, diagnosis, monitoring and treatment protocols. The journal is characterized by the rapid reporting of reviews, original research and clinical studies across all disease areas.

\section{Dovepress}

A key focus is the elucidation of disease processes and management protocols resulting in improved outcomes for the patient.The manuscript management system is completely online and includes a very quick and fair peer-review system. Visit http://www.dovepress.com/ testimonials.php to read real quotes from published authors. 